\title{
Different approaches among physicians to treat pediatric stone disease: a survey-based study
}

\author{
Bülent Önal, M.D., Prof. ${ }^{a}$, Elif A. Kırl, M.D. ${ }^{a}$, Nur Canpolat, M.D. Assoc. Prof. ${ }^{b}$, \\ Mehmet Taşdemir, M.D., Assoc. Prof.', Ahmet Gürbüz, M.D. ${ }^{a}$, Oktay Özman, M.D. ${ }^{a}$, \\ Lale Sever, M.D., Prof. ${ }^{b}$ Ilmay Bilge, M.D., Prof. ${ }^{d}$ and Salim Çalıskan, M.D., Prof. ${ }^{b}$
}

\begin{abstract}
Introduction. Pediatricians, surgeons and subspecialties as pediatric urology and nephrology are involved in the diagnosis and treatment of pediatric renal stone disease (RSD). The aim of this study was to determine diagnostic and treatment approaches, of different disciplines, and to assess differences in their routine diagnostic and treatment protocols.
\end{abstract}

Population and methods. A questionnaire was designed and administered to the participants of the RSD sessions in national congresses of all disciplines in 2017 to evaluate the diagnostic and treatment routines of specialties (surgeons and pediatricians) and subspecialties (pediatric nephrologists and pediatric urologists) for RSD. Results. A total, of 324 questionnaires were analyzed, from 88 pediatricians $(27 \%)$, 121 urologists $(37 \%), 23$ pediatric surgeons $(7 \%), 54$ pediatric nephrologists $(17 \%)$, and 38 pediatric urologists $(12 \%)$. Both groups agreed on the necessity of metabolic evaluation. For distal ureter stones that were $\geq 6 \mathrm{~mm}$; surgeons preferred ureteroscopy (URS), pediatricians preferred shock wave lithotripsy (SWL) $(\mathrm{p}<0.001)$ and subspecialties preferred URS for the treatment $(p=0.636)$. For lower calix stones less than $1 \mathrm{~cm}$ surgeons and subspecialists preferred SWL, while pediatricians preferred hydration $(\mathrm{p}<0.001$, $\mathrm{p}=0.371$ ). For the stone between 1.1 and $2 \mathrm{~cm}$, surgeons preferred intrarenal surgery (RIRS) and SWL, pediatricians preferred SWL $(\mathrm{p}=0.001)$. For larger stones, surgeons and subspecialists preferred percutaneous nephrolithotomy (PCNL), and pediatricians preferred SWL $(p=0.458 p=0.001)$. Pediatric urologist chose low-dose computerized tomography as a diagnostic radiologic evaluation $(\mathrm{p}=0.029)$. Conclusion. There are differences between the disciplines who take an active role in diagnosis and treatment of RSD.

Key words: urolithiasis, child, ureteroscopy, education, therapeutics.

http:/ / dx.doi.org/10.5546/ aap.2021.eng.83

Bülent Önal M.D.

Professor:

bulonal@yahoo.com

Funding:

None.

Conflict of interest:

None.

Received: 5-16-2020

Accepted: 9-10-2020

Onal B, Kirli EA, Canpolat N, Taşdemir et al. Different approaches among physicians to treat pediatric stone disease: a survey-based study. Arch Argent Pediatr 2021;119(2):83-90.

\section{INTRODUCTION}

Although pediatric renal stone disease (RSD) is generally considered a relatively rare disease, it is endemic in some parts of the world. ${ }^{1}$ Pediatricians, surgeons and subspecialties as pediatric urology and nephrology are all involved in the diagnosis and treatment of RSD. ${ }^{1}$ According to our clinical experience although all disciplines play active role in RSD treatment follow the same guidelines, nomograms, and textbooks, differences are observed in daily practice. ${ }^{1-7}$ These differences in diagnostic procedures and treatment approaches by different disciplines have not been assessed yet. However, substantial differences in knowledge and practice can in turn affect the rate of intervention requirements and outcomes.

The aim of this study, was to determine diagnostic and treatment approaches of different disciplines who manage RSD and to assess whether there are differences in their routine diagnostic and treatment protocols.

\section{POPULATION AND METHODS}

After the institutional approval was obtained for this study (number:1456-822) a questionnaire consisting of 13 questions was prepared to answer by physicians from different disciplines who play active roles in the diagnosis, treatment, and follow up of pediatric RSD.

Prior to the preparation of the questions for the survey, the current literature related to pediatric renal stone disease was reviewed and the main issues that differ significantly 
were determined. Two urologists (OÖ, AG), two pediatric urologists (BÖ, EAK) and two pediatric nephrologists $(\mathrm{SC}, \mathrm{NC})$ prepared the questions and scenarios. Before the study, the questions were answered by a group of specialists (pediatricians and surgeons) and subspecialist (pediatric nephrologists and pediatric urologists) with different years of experience in a renal stone disease section of a joint meeting (2017, İstanbul/ Turkey). The authors then reviewed whether the respondents understood and answered the questions.

The questionnaire was administered to the participants of the pediatric RSD sections in National Congress of Urology (2017, Antalya / Turkey), National Congress of Pediatrics (2017, Istanbul/ Turkey) and National Congress of Pediatric Surgery (2017, Antalya/Turkey).

The participants were grouped as pediatricians and surgeons (urologists, pediatric surgeons) for determining the difference between the specialties. Urologists and pediatric surgeons who were officially trained in pediatric urology were considered pediatric urologists. Similarly, pediatricians who were formally trained in pediatric nephrology were considered pediatric nephrologists. Pediatric urologist and nephrologist have common educational programs about RSD (routine joint meetings, journal clubs and lectures) during their fellowship.

The first nine questions aimed to determine the general characteristics of the participants in the two groups, and the remaining questions used classical scenarios that physicians might encounter in their daily routines to assess diagnostic, therapeutic, and follow up methods.

Scenarios were about three different situations related to RSD. First scenario asked the approach to renal microlithiasis (RM) (spot detected $<3 \mathrm{~mm}$ in diameter on renal ultrasound (US) have been described as RM). The other two scenarios were arranged on the different size of stones located in lower calyx and distal ureter.

Completed questionnaires with single response for one question were included; multiple responses for one question were excluded.

Statistical analyses were carried out by SPSS, version 13.0 software (IBM Corp., Armonk, NY, U.S.A.). The Chi-square and Kruskal-Wallis $\mathrm{H}$ test (for multiple group comparison) were used for statistical evaluation. Statistical significance was defined as $\mathrm{p}<0.05$.

TABLE 1. Demographic features of the participants

\begin{tabular}{|c|c|c|c|c|c|}
\hline \multicolumn{6}{|c|}{ Gender, n (\%) } \\
\hline 1. & & \multicolumn{2}{|c|}{ Female } & \multicolumn{2}{|c|}{ Male } \\
\hline Specialties & $\begin{array}{l}\text { Pediatricians } \\
\text { Surgeons }\end{array}$ & \multicolumn{2}{|c|}{$\begin{array}{c}51(58) \\
126(88)\end{array}$} & \multicolumn{2}{|c|}{$\begin{array}{l}37(42) \\
18(12)\end{array}$} \\
\hline \multirow[t]{3}{*}{ Subspecialties } & Pediatric nephrologists & & & & $(48)$ \\
\hline & Pediatric urologists & & & & (45) \\
\hline & & \multicolumn{4}{|c|}{ Current occupation, $\mathrm{n}(\%)$} \\
\hline 2. & & Resident & Fellows & Staff & Senior \\
\hline Specialties & $\begin{array}{l}\text { Pediatricians } \\
\text { Surgeons }\end{array}$ & $\begin{array}{c}15(17) \\
10(7)\end{array}$ & $\begin{array}{l}0(0) \\
0(0)\end{array}$ & $\begin{array}{l}48(54) \\
96(67)\end{array}$ & $\begin{array}{l}25(28) \\
38(26)\end{array}$ \\
\hline \multirow[t]{2}{*}{ Subspecialties } & $\begin{array}{l}\text { Pediatric nephrologists } \\
\text { Pediatric urologists }\end{array}$ & $\begin{array}{l}0(0) \\
0(0)\end{array}$ & $\begin{array}{l}18(33) \\
7(18)\end{array}$ & $\begin{array}{l}16(30) \\
12(32)\end{array}$ & $\begin{array}{l}20(37) \\
19(50)\end{array}$ \\
\hline & & \multicolumn{4}{|c|}{ Year of experience, $\mathrm{n}(\%)$} \\
\hline 3. & & $0-5$ & 6-10 & $11-15$ & $\geq 15$ \\
\hline Specialties & $\begin{array}{l}\text { Pediatricians } \\
\text { Surgeons }\end{array}$ & $\begin{array}{l}17(19) \\
12(8)\end{array}$ & $\begin{array}{l}22(25) \\
66(46)\end{array}$ & $\begin{array}{l}21(24) \\
21(15)\end{array}$ & $\begin{array}{l}28(32) \\
45(31)\end{array}$ \\
\hline \multirow[t]{2}{*}{ Subspecialties } & $\begin{array}{l}\text { Pediatric nephrologists } \\
\text { Pediatric urologists }\end{array}$ & $\begin{array}{l}7(13) \\
4(11)\end{array}$ & $\begin{array}{c}11(20) \\
3(8)\end{array}$ & $\begin{array}{l}16(30) \\
12(32)\end{array}$ & $\begin{array}{l}20(37) \\
19(50)\end{array}$ \\
\hline & & \multicolumn{4}{|c|}{ Institution, $\mathrm{n}(\%)$} \\
\hline 4. & & University & & sspital & Private practice \\
\hline Specialties & $\begin{array}{l}\text { Pediatricians } \\
\text { Surgeons }\end{array}$ & $\begin{array}{l}49(56) \\
73(51)\end{array}$ & & & $\begin{array}{l}2(2) \\
10(7)\end{array}$ \\
\hline Subspecialties & $\begin{array}{l}\text { Pediatric nephrologists } \\
\text { Pediatric urologists }\end{array}$ & $\begin{array}{l}40(74) \\
20(53)\end{array}$ & & & $\begin{array}{l}0(0) \\
7(18)\end{array}$ \\
\hline
\end{tabular}




\section{RESULTS}

A total, of 348 physicians participated in the survey, and after exclusion, 324 questionnaires were analyzed. The specialists were 88 pediatricians $(27 \%), 121$ urologists $(37 \%)$, 23 pediatric surgeons $(7 \%)$. The subspecialists were 54 pediatric nephrologists $(17 \%)$, and 38 pediatric urologists (12\%) (Figure 1). Specialties were divided into two groups: pediatricians $(n=88)$ and surgeons $(n=144)$; their responses were evaluated based on their specializations. Subspecialties' answers were also compared. The demographic characteristics of the participants are summarized in Table 1.

\section{Questions for metabolic evaluations}

Although the evaluation workups of participants varied in daily routine, the specialties and subspecialties agreed on the necessity of metabolic evaluation. While pediatricians performed metabolic evaluation more than the surgeons ( $83 \%$ vs. $53 \%$ ), this rate was similar between subspecialists (98\% vs. $95 \%$ ). More surgeons preferred the 24-hour urine test to two spot urine analyses, as compared to pediatricians (44\% vs. $66 \%$, p: 0.032). Subspecialties agreed on performing 24 -hour urine test (72\% vs. $76 \%$, p: 0.299). The participants who did not perform metabolic evaluation indicated the most lack of education as a reason. Moreover, pediatricians recommended children with RSD should be followed by pediatric nephrologists ( $52 \%$ vs. $19 \%$, p: 0.001), while the subspecialties agreed that a multidisciplinary team should follow up (73\% vs. $74 \%$, p: 0.657) (Table 2).

\section{Questions about clinical scenarios}

Participants were asked in terms of their approaches to infantile RM. The majority of specialists preferred not to perform the sodium nitroprusside test in the evaluation of RM $(87 \%$ vs. $81 \%$, p: 0.113 ). In contrast, subspecialists agree on nitroprusside test in evaluation of RM (85\% vs. $74 \%$, p: 0.252). Radiological evaluation for RM did not differ between the groups, and US was the most preferred tool for both specialties (76 \% vs. $67 \%$, p: 0.09$)$ and subspecialties (83\% vs. $79 \%$, p: 0.672 ). Both groups agreed on treatment modalities, and the rates of follow up with US and follow up with hydration were almost identical (Table 3).

The participants were also asked about their approach to symptomatic non-complicated distal ureter stones. Most of participants in both groups chose a similar procedure for distal ureter stones that are 3 to $5 \mathrm{~mm}$ in diameter. The specialists differed in their treatment options for distal ureter stones that are $\geq 6 \mathrm{~mm}$; while surgeons (52\%) preferred ureteroscopy (URS), pediatricians $(36 \%)$ preferred shock wave lithotripsy (SWL) $(\mathrm{p}<0.001 ;$ Table 4). However, subspecialists both preferred URS for the treatment (66\% vs. $68 \%$, p: 0.636).

Participants were asked about the treatment of lower calix stones of different sizes. If a stone was less than $1 \mathrm{~cm}$, the first choice for surgeons was SWL, while that for pediatricians was hydration (54\% vs. $40 \%$, p < 0.001). Subspecialists both preferred SWL for the first line treatment (55\% vs. $55 \%$, p: 0.371). If a stone was between 1.1 and

\section{FIGURE 1. The flowchart diagram of participants}

Total number of participants invited for survey in national congress $n=420$.

Urologist $n=130$, pediatrician $n=130$, pediatric surgeon $n=60$,

pediatric nephrologist $n=60$, pediatric urologist $n=40$.

Total number of participants accepted participation $\mathrm{n}=348$.

Urologist $\mathrm{n}=129$, pediatrician $\mathrm{n}=92$, pediatric surgeon $\mathrm{n}=35$, pediatric nephrologist $\mathrm{n}=58$, pediatric urologist $\mathrm{n}=40$.

Total number of eliminated surveys $\mathrm{n}=24$.

Urologist $\mathrm{n}=8$, pediatrician $\mathrm{n}=4$, pediatric surgeon $\mathrm{n}=12$, pediatric nephrologist $\mathrm{n}=4$, pediatric urologist $\mathrm{n}=2$.

Urologist $\mathrm{n}=121$, pediatrician $\mathrm{n}=88$, pediatric surgeon $\mathrm{n}=23$, pediatric nephrologist $\mathrm{n}=54$, pediatric urologist $\mathrm{n}=38$. 
TABLE 2. Approach to diagnostic workup between the specialties

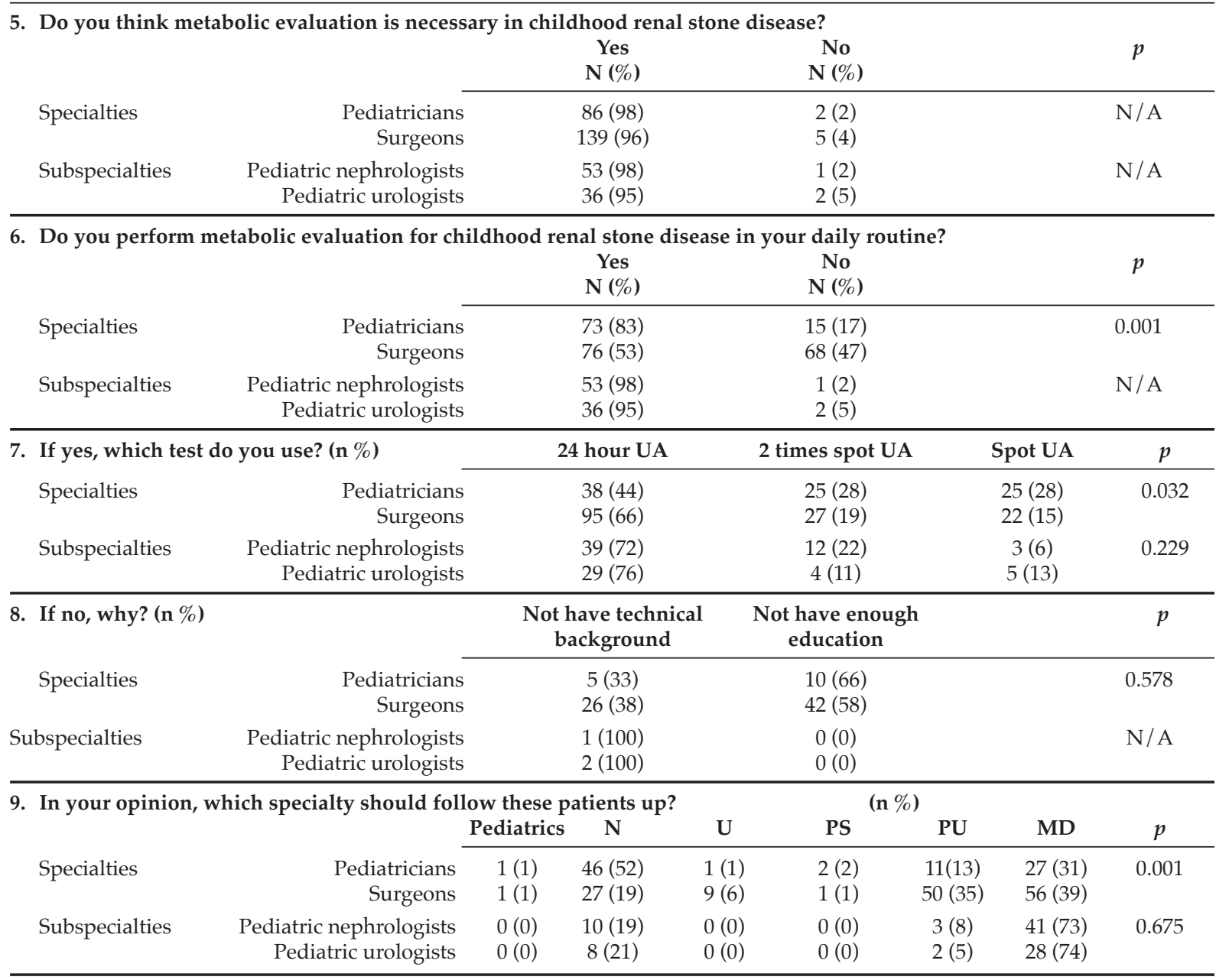

MD:Multidisciplinary N: nephrologist PS: pediatric surgeon PU: pediatric urologist U: urologist UA: urine analysis N/A: not available.

TABLE 3. Approach to evaluation and treatment modality of renal microlithiasis (RM) between the specialties

\begin{tabular}{|c|c|c|c|c|c|c|c|}
\hline \multicolumn{4}{|c|}{ 10a Do you evaluate this baby with urine sodium nitroprusside test? } & \multirow{2}{*}{$\begin{array}{c}\text { Yes, n (\%) } \\
11(13) \\
27(19)\end{array}$} & \multirow{2}{*}{\multicolumn{2}{|c|}{$\begin{array}{c}\text { No, n (\%) } \\
77(87) \\
117(81)\end{array}$}} & \multirow{2}{*}{$\begin{array}{c}p \\
0.113\end{array}$} \\
\hline Specialties & \multicolumn{2}{|l|}{$\begin{array}{r}\text { Pediatricians } \\
\text { Surgeons }\end{array}$} & & & & & \\
\hline Subspecialties & $\begin{array}{r}\text { Pediatric nephrologists } \\
\text { Pediatric urologists }\end{array}$ & & & $\begin{array}{l}46(85) \\
29(74)\end{array}$ & \multicolumn{2}{|c|}{$\begin{array}{l}8(15) \\
9(26)\end{array}$} & 0.252 \\
\hline \multicolumn{2}{|c|}{ 10b Which radiological method do you prefer next? } & $\mathrm{CT}$ & IVP & $\begin{array}{l}\text { DUSG } \\
\text { US }\end{array}$ & US & DUSG & $p$ \\
\hline Specialties & $\begin{array}{r}\text { Pediatricians } \\
\text { Surgeons }\end{array}$ & $\begin{array}{l}4(3) \\
13(7)\end{array}$ & $\begin{array}{l}1(1) \\
5(3)\end{array}$ & $\begin{array}{l}26(18) \\
38(21)\end{array}$ & $\begin{array}{l}3(2) \\
4(2)\end{array}$ & $\begin{array}{l}108(76) \\
122(67)\end{array}$ & 0.09 \\
\hline Subspecialties & $\begin{array}{r}\text { Pediatric nephrologists } \\
\text { Pediatric urologists }\end{array}$ & $\begin{array}{l}0(0) \\
0(0)\end{array}$ & $\begin{array}{l}0(0) \\
0(0)\end{array}$ & $\begin{array}{l}9(17) \\
8(21)\end{array}$ & $\begin{array}{l}0(0) \\
0(0)\end{array}$ & $\begin{array}{l}45(83) \\
30(79)\end{array}$ & 0.672 \\
\hline 10c Which treatmen & odality do you prefer for this baby? & $\begin{array}{l}\text { Surgical } \\
\text { approach }\end{array}$ & $\begin{array}{c}\text { USG } \\
\text { Follow-up }\end{array}$ & $\begin{array}{l}\text { Hydration } \\
\text { Follow-up }\end{array}$ & Multi & Ignore & $p$ \\
\hline Specialties & $\begin{array}{r}\text { Pediatricians } \\
\text { Surgeons }\end{array}$ & $\begin{array}{l}1(1) \\
6(4)\end{array}$ & $\begin{array}{l}42(48) \\
70(49)\end{array}$ & $\begin{array}{l}36(41) \\
53(37)\end{array}$ & $\begin{array}{c}8(9) \\
14(10)\end{array}$ & $\begin{array}{l}1(1) \\
1(1)\end{array}$ & 0.956 \\
\hline Subspecialties & $\begin{array}{l}\text { Pediatric nephrology } \\
\text { Pediatric urologists }\end{array}$ & $\begin{array}{l}0(0) \\
0(0)\end{array}$ & $\begin{array}{l}25(46) \\
17(45)\end{array}$ & $\begin{array}{l}24(44) \\
19(50)\end{array}$ & $\begin{array}{l}5(9) \\
2(5)\end{array}$ & $\begin{array}{l}0(0) \\
0(0)\end{array}$ & 0.732 \\
\hline
\end{tabular}

CT: computed tomography; DUSG: direct urinary system graphy; IVP: intravenous pyelography; US: ultrasonography. 
$2 \mathrm{~cm}$, surgeons preferred intrarenal surgery (RIRS) $(33 \%)$ and SWL (33 \%) almost equally, but most of pediatricians preferred SWL (40\%), (p: 0.001). If a stone was larger than $2 \mathrm{~cm}$, most of surgeons preferred percutaneous nephrolithotomy (PCNL) $(78 \%)$, and pediatricians preferred SWL $(51 \%)$ as a first option and PCNL (33\%) as a second option (p: 0.001). Subspecialists preferred PCNL for the treatment (56\% vs. $63 \%$, p: 0.458).

This difference was also seen in the selection of radiological imaging methods. Participants of both specialties preferred plain film kidney, ureter, and bladder (KUB) radiography plus US evaluation rather than US alone. However, half of the pediatric urologist (53\%) chose low-dose computerized tomography (CT) and pediatric nephrologists chose DUSG and US (46\%) as a diagnostic tool (p: 0.029) (Table 5).

\section{DISCUSSION}

The management of pediatric RSD involves multiple disciplines. Previous studies in the literature have mostly focused on diagnostic approaches, medical and surgical treatment options that improve outcomes. The present study however focused on the differences in diagnostic and treatment approaches by different disciplines. The remarkable result of the study shows that pediatricians and surgeons may have different diagnostic and therapeutic approaches. Another notable implication of this study is that pediatric nephrologist and urologist adopt similar diagnostic and therapeutic approaches associated with RSD.

Most participants in this study have more than 5 years of experience, implying that they know the existing guidelines on the management of RSD and therefore are equipped to handle complicated urinary stones.

According to current guidelines, due to the high incidence of predisposing factors and high stone recurrence rates, every child with a urinary stone should undergo complete metabolic evaluation. . $^{1,3,6}$ Despite the differences in the daily practice, all participants agreed on the importance of metabolic evaluation. Although the pediatricians perform metabolic evaluation more frequently than the surgeons do, they prefer spot urine analysis. Subspecialists and surgeons preferred the 24-hour urine test. Spot urine analysis is easy to perform in children, but the results can be misleading. This orientation will lead to increased diagnostic complexity. An important point indicated by the specialties is lack of capacitation during their training, as reason for omitting to perform metabolic evaluation, however, for the subspecialties not having enough technical background is the only reason not to perform the

TABLE 4. Diagnostic and treatment modalities for different stone sizes in distal ureter

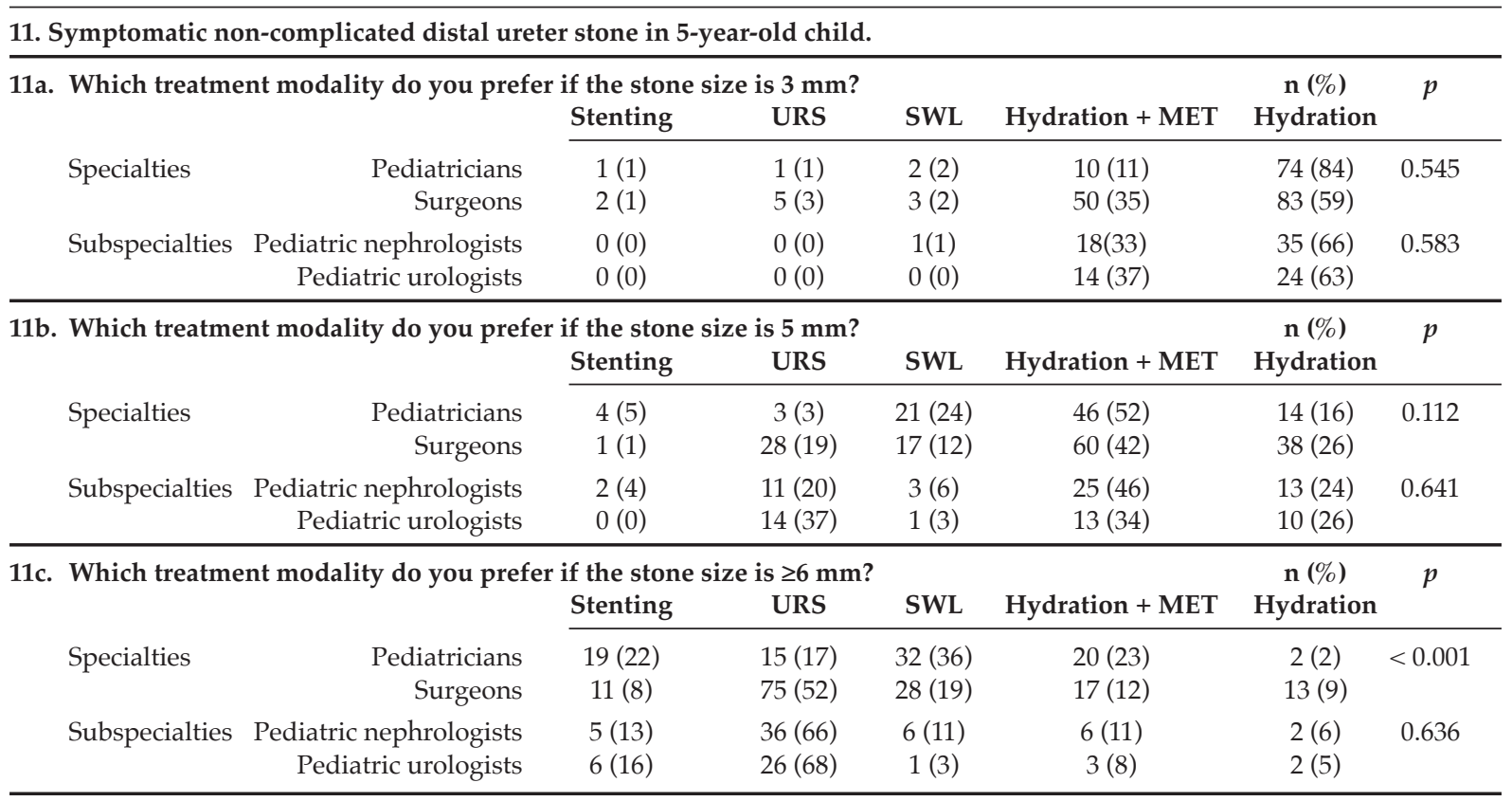

MET: medical expulsive therapy; URS: ureteroscopy; SWL: shock wave lithotripsy. 
metabolic tests. Additionally, the availability of diagnostic methods and treatments, which could vary between the public and private sectors, and consequently influence the decision-making process when choosing or preferring one or another method. All reasons are the risk factors for delay diagnosis of underlying metabolic disorder.

There are few reports on the diagnosis, evaluation, management, long-term follow up, and outcomes of RM in the literature. Due to the lack of controlled studies, the real incidence, and the steps to be followed in diagnosis and treatment are unclear. ${ }^{8,9}$ This lack of clarity was demonstrated in our study, as both groups preferred different diagnostic evaluations and conservative management, such as hydration and close follow up with USG, for RM. Most of pediatric patients with RM have one or more underlying metabolic disorders which are similar to those with renal stones. ${ }^{8,9}$ Although subspecialists agreed on performing sodium nitroprusside test is useful for the first-line metabolic evaluation, specialists agreed on there is no requirement of metabolic evaluation. This result may be alarming because this subject has not been sufficiently clarified in the literature and requires physicians to be careful.

Medical expulsive therapy (MET), mostly with alpha-blockers, was recommended for uncomplicated distal ureteral stones $<10 \mathrm{~mm} .{ }^{5}$ As a reflection of this, participants agreed on MET and hydration treatment modalities for small distal ureteral stones although there are conflicts in the literature about off-label use of MET. ${ }^{10}$ The specialists also differed in their treatment approaches for distal ureter stones that are $\geq 6 \mathrm{~mm}$. SWL has been used to treat ureteral calculi with lower success rates for distal ureteric stones, and pediatricians tend to insist on SWL treatment or conservative management; however, surgeons and both subspecialists prefer URS. This orientation is correlated with the clinical guidelines. ${ }^{1,11,12}$

TABLE 5. Diagnostic and treatment modalities for different stone size in renal calyx

\begin{tabular}{|c|c|c|c|c|c|c|c|c|}
\hline \multirow[t]{6}{*}{ 12a. } & \multicolumn{8}{|c|}{ Which treatment modality do you prefer if the stone size is under $1 \mathrm{~cm}$ ? } \\
\hline & & & PCNL & RIRS & SWL & Hydration MET & Hydration & $p$ \\
\hline & \multirow[t]{2}{*}{ Specialties } & Pediatricians & $2(2)$ & $3(3)$ & $13(15)$ & $23(26)$ & $47(54)$ & \multirow[t]{2}{*}{0.001} \\
\hline & & Surgeons & $1(1)$ & $14(10)$ & $57(40)$ & $26(18)$ & $46(32)$ & \\
\hline & \multirow[t]{2}{*}{ Subspecialties } & Pediatric nephrologists & $2(4)$ & $1(2)$ & $30(55)$ & $11(20)$ & $10(19)$ & \multirow[t]{2}{*}{0,371} \\
\hline & & Pediatric urologists & $3(8)$ & $5(13)$ & $21(55)$ & $4(11)$ & $5(13)$ & \\
\hline \multirow[t]{6}{*}{$12 b$. } & \multicolumn{6}{|c|}{ Which treatment modality do you prefer if the stone size is between 1.1 and $2 \mathrm{~cm}$ ? } & n $(\%)$ & \multirow[b]{2}{*}{$p$} \\
\hline & & & PCNL & RIRS & SWL & Hydration MET & Hydration & \\
\hline & \multirow[t]{2}{*}{ Specialties } & Pediatricians & $7(8)$ & $9(10)$ & $35(40)$ & $32(36)$ & $5(6)$ & \multirow[t]{2}{*}{0.001} \\
\hline & & Surgeons & $36(25)$ & $48(33)$ & $44(31)$ & $12(8)$ & $4(3)$ & \\
\hline & \multirow[t]{2}{*}{ Subspecialties } & Pediatric nephrologists & $7(13)$ & $7(13)$ & $33(61)$ & $5(9)$ & $2(4)$ & \multirow[t]{2}{*}{0.246} \\
\hline & & Pediatric urologists & $6(16)$ & $7(18)$ & $23(60)$ & $1(3)$ & $1(3)$ & \\
\hline \multirow[t]{6}{*}{ 12c. } & \multicolumn{6}{|c|}{ Which treatment modality do you prefer if the stone size is above $2 \mathrm{~cm}$ ? } & n $(\%)$ & \multirow[b]{2}{*}{$p$} \\
\hline & & & PCNL & RIRS & SWL & Hydration MET & Hydration & \\
\hline & \multirow[t]{2}{*}{ Specialties } & Pediatricians & $29(33)$ & $8(9)$ & $45(51)$ & $4(5)$ & $2(2)$ & \multirow[t]{2}{*}{0.001} \\
\hline & & Surgeons & $112(78)$ & $17(12)$ & $14(9)$ & $2(1)$ & $0(0)$ & \\
\hline & \multirow[t]{2}{*}{ Subspecialties } & Pediatric nephrologists & $30(56)$ & $12(19)$ & $10(22)$ & $2(2)$ & $0(0)$ & \multirow[t]{2}{*}{0.458} \\
\hline & & Pediatric urologists & $24(63)$ & $10(26)$ & $4(11)$ & $0(0)$ & $0(0)$ & \\
\hline \multirow[t]{6}{*}{13.} & \multicolumn{8}{|c|}{ 10-year-old child admitted to ER with the complaint of renal colic. Which imaging method do you prefer? n (\%) } \\
\hline & & & CT & DUSG US & DUSG & US & & $p$ \\
\hline & \multirow[t]{2}{*}{ Specialties } & Pediatricians & $4(5)$ & $42(48)$ & $3(3)$ & $39(44)$ & & \multirow[t]{2}{*}{0.057} \\
\hline & & Surgeons & $26(18)$ & $68(47)$ & $1(1)$ & $49(34)$ & & \\
\hline & \multirow[t]{2}{*}{ Subspecialties } & Pediatric nephrologists & $5(9)$ & $25(46)$ & $3(6)$ & $21(39)$ & & \multirow[t]{2}{*}{0.029} \\
\hline & & Pediatric urologists & $20(53)$ & $9(24)$ & $2(5)$ & $7(18)$ & & \\
\hline
\end{tabular}

CT: computerized tomography; DUSG: direct urinary system graphy; ER: emergency room; MET: medical expulsive therapy; PCNL: percutaneous nephrolithotomy; RIRS: retrograde intrarenal surgery; URS: ureteroscopy; US: ultrasonography. 
Although SWL is recommended as a first-line treatment modality for caliceal stones smaller than $10 \mathrm{~mm},{ }^{1}$ pediatricians prefer hydration therapy, while surgeons and subspecialists prefer SWL. This approach can explain by SWL was not completely accepted as a noninvasive treatment by pediatricians due to the need for sedo-analgesia or general anesthesia in children. RIRS has recently become more popular due to its higher stone-free rate compared to SWL.,13 Due to fact that surgeons preferred RIRS (33\%) and SWL (31 \%) almost equally for the treatment of stone sized 1.1 and $2 \mathrm{~cm}$. Most of surgeons and subspecialists preferred PCNL, but pediatricians preferred still SWL as a first option for stones larger than $2 \mathrm{~cm}$. This observation can be explained by the fact that pediatricians tend to act more conservatively than surgeons. Another possible explanation is that most of pediatricians are not familiar enough with these minimally invasive procedures.

Specialists and pediatric nephrologists equally preferred USG and/or KUB for children presenting with renal colic. This result could be secondary to following an as low as reasonably achievable (ALARA) protocol in children. ${ }^{14}$ Recently, some studies reported that low-dose CT scan was better than USG at detecting stone and qualifying renal anatomy. ${ }^{15}$ Therefore, half of pediatric urologists prefer to use low-dose CT in diagnosis. This preference among surgeons may be because surgeons want to observe the renal anatomy and stone burden and location, in order to facilitate the surgical procedure.

Although pediatricians recommended children with RSD should be followed by pediatric nephrologist, the subspecialists agreed that a multidisciplinary approach involving more specialized disciplines, such as pediatric urology and nephrology should follow up these patients.

In summary, departments treating RSD may have different approaches related to diagnosis and the treatment protocols. This difference may be related with the lack of evidence or appropriate guidelines and the rapid evolution of technology. Additionally, the inherent conflict of interest that a specialist might have to prefer a certain diagnostic and therapeutic modality may be another reason of the different choses. It may also be related to caseload. A larger number of patients with stones lead to better experience and familiarity with current literature. In this survey, subspecialties gave similar answers to clinical scenarios and questions about the metabolic evaluation. This similarity may be the result of common educational programs provides the integrity of these disciplines in the management of pediatric RSD and lead them to work as a stone-team.

One of the limitations of this study is that the data were obtained from a multiple-choice questionnaire, and participants were forced to adapt their ideas to the options that were prepared in simplified forms. Therefore, the results may not perfectly reflect their daily practices. Another limitation is that our questionnaire was not validated, which may create potential bias for responders. Additionally, the findings were only from respondents available to the authors. They may be representative of the study population, but it may not be appropriate to generalize the results of the study to other populations. Despite these limitations, our results reveal the differences in diagnostic, treatment, and follow up approaches of physicians from different disciplines who play active roles in pediatric RSD. The preference of different diagnostic and treatment modalities across disciplines may affect diagnostic procedures and treatment modalities and result in lower stone-free rate and increased recurrence rate.

\section{CONCLUSION}

There are differences between the disciplines who take an active role in diagnosis and treatment of RSD.

The integration of disciplines in the management of pediatric RSD and generating a stone teams may lead to decrease the conflicts in diagnostic and treatment approaches.

\section{REFERENCES}

1. Tekgül S, Doğan HS, Hoebeke P, Kocvara R, et al. Urinary stone disease. In: EAU Guidelines on Pediatric Urology. 2016;58-65. [Accessed on: Sept 10, 2020]. Available in: https: / / uroweb.org/wp-content/uploads / EAUGuidelines-Paediatric-Urology-2016.pdf.

2. Assimos D, Krambeck A, Miller N.L, Monga M, et al. Surgical management of stones: American Urological Association/ Endourological Society guideline. Part I. J Urol. 2016; 196(4):1153-60.

3. Radmayr C, Bogaert G, Doğan HS, Nijman JM, et al. Urinary Stone disease. In: EAU Guidelines on pediatric Urology. 2020: 65-74. [Accessed on: Sept 10, 2020]. Available in: https: / / uroweb.org/guideline/paediatric-urology /

4. SchneckFX, OstM.Surgical management of pediatric stone disease. In: Wein AJ, Kavoussi LR, Partin AW, Peter CA. Cambell-Walsh Urology. 11th ed. Philadelphia: Elsevier; 2016.p.3102-20.

5. Türk C, Petrik A, Sarica K, Seitz C et al. EAU guidelines on diagnosis and conservative management of urolithiasis. Eur Urol. 2016; 69(3):468-74. 
6. Straub, M. Strohmaier WL, Berg W, Beck B et al. Diagnosis and metaphylaxis of stone disease. Consensus concept of the National Working Committee on Stone Disease for the upcoming German Urolithiasis Guideline. World J Urol. 2005; 23(5):309-23.

7. Liu W, Esler SJ, Kenny BJ, Goh RH, et al. Low-dose nonenhanced helical CT of renal colic: assessment of ureteric stone detection and measurement of effective dose equivalent. Radiology. 2015; (1):51-4.

8. Fallahzade MA, Hassanzadeh J, Fallahzadeh MH. What do we know about pediatric renal microlithiasis? J Renal Inj Prev. 2017; (6):70-5.

9. Bilge I, Yılmaz A, Kayıran SM, Emre S, et al. Clinical importance of renal calyceal microlithiasis in children. Pediatr Int. 2013; 55(6):731-6.

10. Knoll T, Türk C. The role of medical expulsive therapy for ureteral stones: pro MET. Eur Urol Focus. 2017; 3(1):3-4.

11. Onal, B, Demirkesen O, Tansu N, Kalkan M, et al. The impact of caliceal pelvic anatomy on stone clearance after shock wave lithotripsy for pediatric lower pole stones. $J$ Urol. 2004; 172(3):1082-6.

12. Hochreiter WW, Danuser H, Perrig M, Studer UE. Extracorporeal shock wave lithotripsy for distal ureteral calculi: what a powerful machine can achieve. J Urol. 2003; 169(3):878-80.

13. Ateş F, Zor M, Yılmaz O, Tuncer M, et al. Management behaviors of the urology practitioners to the small lower calyceal stones: the result of a web-based survey. Urolithiasis. 2016; 44(3):277-81

14. Sodhi KS, Krishna S, Saxena AK, Sinha A, et al. Clinical application of 'justification' and 'optimization' principle of ALARA in pediatric CT imaging: "How many children can be protected from unnecessary radiation?" Eur J Radiol. 2015; 84(9):1752-7.

15. Passerotti C, Chow JS, Silva A, Schoettler CL, et al. Ultrasound versus computerized tomography for evaluating urolithiasis. J Urol. 2009; 182(4 Suppl):1829-34. 